\title{
I-123 metaiodobenzylguanidine innervation imaging as a tool for norepinephrine transporter research: A possible application for genetic analysis in heart failure
}

\author{
Kenichi Nakajima, $\mathrm{MD}, \mathrm{PhD},{ }^{\mathrm{a}}$ Ichiro Matsunari, $\mathrm{MD}, \mathrm{PhD},{ }^{\mathrm{b}}$ and \\ Arnold F. Jacobson, MD, PhD \\ a Department of Nuclear Medicine, Kanazawa University, Kanazawa, Japan \\ b Division of Nuclear Medicine, Department of Radiology, Saitama Medical University Hospital, \\ Saitama, Japan \\ c Diagram Consulting, Kihei, HI
}

Received Nov 14, 2016; accepted Nov 14, 2016

doi: $10.1007 /$ s12350-016-0739-1

\section{See related article, pp. 900-906}

Nuclear cardiology is widely used to characterize the physiologic condition of the muscles, blood vessels, and sympathetic nerves of the heart. Myocardial perfusion imaging, in combination with functional study with electrocardiographic gating, allows detection of myocardial ischemia and infarction in patients with coronary artery disease. While nuclear imaging evaluates perfusion at the myocardial cellular level, x-ray computed tomography and magnetic resonance imaging can be used to assess perfusion, and provide visualization of the coronary vascular anatomy responsible for different patterns of myocardial blood flow. However, for examining the status of myocardial sympathetic nerves, nuclear methods using the SPECT agent ${ }^{123} \mathrm{I}-$ mIBG or positron tracers such as ${ }^{11} \mathrm{C}$-hydroxyephedrine, ${ }^{1}{ }^{11} \mathrm{C}$-epinephrine, ${ }^{2}{ }^{11} \mathrm{C}$-CGP12177, ${ }^{3}$ and ${ }^{11} \mathrm{C}-\mathrm{Me} @$ HAPTHI (preclinical) ${ }^{4}$ provide unique information not available using anatomic imaging modalities.

Editorial for the Journal of Nuclear Cardiology on: Derk Verschure, et al. Polymorphism of SLC6A2 gene does not influence outcome of myocardial ${ }^{123} \mathrm{I}$-mIBG scintigraphy in patients with chronic heart failure.

Reprint requests: Kenichi Nakajima, MD, PhD, Department of Nuclear Medicine, Kanazawa University, Kanazawa, 920-8641, Japan; nakajima@med.kanazawa-u.ac.jp

J Nucl Cardiol 2018;25:907-10.

$1071-3581 / \$ 34.00$

Copyright (C) 2016 American Society of Nuclear Cardiology.

\section{${ }^{123}$ I-MIBG UPTAKE MECHANISM}

Norepinephrine transporter (NET) is responsible for reuptake of extra-cellular norepinephrine (NE) and dopamine, and plays an essential role for regulating sympathetic nervous transmission and function. Among the imaging agents available for studying the NET system, ${ }^{123} \mathrm{I}-\mathrm{mIBG}$, an analogue of NE, has been the most successfully utilized. ${ }^{123} \mathrm{I}-\mathrm{mIBG}$ is taken up into the synaptic terminal primarily via NET-mediated reuptake (uptake 1), stored in synaptic vesicles, and released into synaptic clefts. Due to the different structure from norepinephrine, ${ }^{123} \mathrm{I}-\mathrm{mIBG}$ does not bind to post-synaptic receptors and is not metabolized by monoamine oxidase or catechol-O-methyl transferase. However, as ${ }^{123} \mathrm{I}-\mathrm{mIBG}$ and norepinephrine share the same uptake mechanism, the hypothesis that variation in the gene which codes for the NET system might influence ${ }^{123} \mathrm{I}$ mIBG imaging findings, as explored in the study by Verschure et al, is understandable. ${ }^{5}$

\section{${ }^{123}$ I-MIBG IMAGING AS A CLINICAL TOOL}

One of the major indications for ${ }^{123} \mathrm{I}-\mathrm{mIBG}$ imaging is heart failure (HF). In Japan, after official approval of ${ }^{123}$ I-mIBG (MyoMIBG, FUJIFILM RI Pharma, Co. Ltd) during 1992,6 diagnostic and prognostic use of ${ }^{123} \mathrm{I}$ mIBG is a class I indication, and use for examining the effect of medications is a class IIa indication. ${ }^{7}$ In the USA, the indication for ${ }^{123} \mathrm{I}-\mathrm{mIBG}$ (Adreview, GE Healthcare) is for risk assessment of patients with $\mathrm{HF}^{8}$ with New York Heart Association functional classes II and III, based on the ADMIRE-HF multicenter study. ${ }^{9}$ 
Table 1. Areas of research and clinical interest regarding ${ }^{123} \mathrm{I}$-mIBG and NET system

Physiology, pathophysiology

Development of new radiotracers for NET system

Genetics of NET

Appropriate kinetic model

Age and gender dependency of ${ }^{123} \mathrm{I}$-mIBG

Effect of medications on ${ }^{123} \mathrm{I}$-mIBG parameters

Clinical applications of ${ }^{123} \mathrm{I}$-mIBG in cardiology

Coronary artery disease

Cardiomyopathies

Arrhythmogenicity

Heart failure, diagnosis, and prognosis

Treatment effects by medications

Indications of cardiac devices for lethal arrhythmia

Chemotherapy and drug-induced cardiotoxicity

Denervation/reinnervation

Clinical applications of ${ }^{123}$ I-mIBG in neurology

Lewy body diseases including Parkinson disease,

dementia with Lewy bodies, and pure autonomic

failure

$N E T$ norepinephrine transporter

In Europe, cardiac indications for ${ }^{123}$ I-mIBG vary by country and often do not specifically refer to HF patients.

Other cardiac utilizations of ${ }^{123}$ I-mIBG imaging besides for HF, for both physiological and pathological conditions, are listed in Table 1. In Japan, since indications of ${ }^{123} \mathrm{I}$-mIBG were widely approved in cardiac diseases, various applications of both clinical and pathophysiological bases have been investigated. The most effective application was cardiac diseases related to ischemia, which included acute and subacute phase of infarction, ${ }^{10}$ coronary spastic angina, and unstable angina. Neurological application for Lewy body disease is also widely used, which included Parkinson disease, dementia with Lewy body disease (DLB) and pure autonomic failure. ${ }^{11,12}$ Since prodromal DLB and diagnostic overlap with Alzheimer disease and DLB are also known to exist, diagnostic ability of ${ }^{123}$ I-mIBG in such atypical cases has been noted. ${ }^{13}$

\section{SIGNIFICANCE OF REDUCED ${ }^{123}$ I-MIBG UPTAKE}

As decrease in ${ }^{123}$ I-mIBG uptake is observed in a wide variety of pathophysiological conditions, does ${ }^{123} \mathrm{I}$ mIBG reflect results of secondary myocardial damage and dysfunction or any cause of diseases? Sympathetic activity suppression may be seen in spastic angina ${ }^{14}$ and Takotsubo cardiomyopathy, but imaging defects could be the result of ischemic condition at a microcirculation level. In conditions when transmural or scattered fibrosis occurs, even in infarction and cardiomyopathies, both regional myocardial perfusion and innervation are affected. Sympathetic defect on ${ }^{123} \mathrm{I}$-mIBG images is always larger than perfusion defect, and the regional mismatch may be related to the occurrence of ventricular tachycardia or lethal arrhythmogenicity. ${ }^{15}$ As such, although the pathophysiological meaning of derangement of activity with respect to causal relationship remains unknown, abnormal ${ }^{123} \mathrm{I}-\mathrm{mIBG}$ activity in the heart is a sensitive and sometimes early finding to identify NE transporter abnormality. Even when genetic influence on NET system is demonstrated in patients with $\mathrm{HF}$, secondary myocardial damage unrelated to genetic abnormalities may coexist. In addition, contribution of non-specific ${ }^{123} \mathrm{I}$-mIBG uptake (non-NETmediated) on early and late imaging findings is not well understood. Therefore, careful differentiation between a true genetic effect and HF-related myocardial damage will be required.

\section{${ }^{123}$ I-MIBG AND RESEARCH ON NET}

Verschure et al evaluated the NET (uptake-1), encoded by the solute carrier family 6 (SLC6A2), ${ }^{5}$ in a series of HF patients undergoing ${ }^{123}$ I-mIBG imaging. Although several single-nucleotide polymorphisms (SNP) were found in their study, none of the SNPs were functional. Thus, it is not surprising that no associations were found between these polymorphisms and any ${ }^{123} \mathrm{I}$-mIBG parameters of cardiac sympathetic activity such as heart-to-mediastinum (H/M) ratio or washout rate. Functional variations in NET SNPs are known to exist in psychiatric and cardiovascular phenotypes such as idiopathic postural orthostatic intolerance, and therapeutic applications for regulating NET system have been sought in attention-deficit hyperactivity disorder, neurodegenerative disorders, depressive disorders, and other psychiatric diseases. ${ }^{16,17}$ However, the likelihood that such variants would be found in a broad heterogeneous population of $\mathrm{HF}$ patients is small. The question of whether presence of functional NET SNPs might play a role in development or progression of $\mathrm{HF}$, and thereby be detectable using functional imaging such as with ${ }^{123} \mathrm{I}-\mathrm{mIBG}$, remains unanswered. Even if there is a connection between NET SNPs and HF pathophysiologic condition, it would probably require genomic analysis of thousands of patients to characterize the association. Given the complex pathophysiological factors that influence cardiac ${ }^{123} \mathrm{I}$-mIBG kinetics, such as storage, release, and reuptake, much more work to elucidate genetic influences on cardiac uptake of ${ }^{123} \mathrm{I}$-mIBG is needed. 


\section{EXPECTATION FOR TECHNOLOGICAL IMPROVEMENTS IN TRACER KINETICS}

The study of Verschure et al represents only a starting point for examining the relationships between NET genetics in HF and ${ }^{123} \mathrm{I}$-mIBG kinetics. The H/M ratio is a very crude parameter to analyze whole-heart kinetics of MIBG, although it is a practical tool for clinical evaluation of risk in HF. Standardization of methodology for calculating $\mathrm{H} / \mathrm{M}$ ratio improves its usefulness with different camera systems and risk model creation. ${ }^{18,19}$ Nevertheless, more precise analysis using positron tracers allows determination of parameters such as receptor binding capacity, rate constants, and volume of reaction as in cardiac beta-adrenergic receptor study with ${ }^{11} \mathrm{C}$-CGP $12177 .{ }^{3}$ Analysis using ${ }^{11} \mathrm{C}$-hydroxyephedrine uses parameters of clearance or retention parameters. ${ }^{1}$ Improvements in ${ }^{123} \mathrm{I}-\mathrm{mIBG}$ imaging are possible using high-resolution and high sensitivity cadmium-zinc-telluride cameras, which permit dynamic SPECT acquisitions. ${ }^{20}$ Further, absolute quantification with a unit of $\mathrm{Bq} / \mathrm{cm}^{3}$ is feasible using SPECT/CT. Appropriate kinetic modeling is also indispensable for analyzing kinetics around the sympathetic terminal. New radiotracers such as ${ }^{11} \mathrm{C}$-Me@HAPTHI offer promise for better demonstration of the NET system, ${ }^{4}$ and these preclinical investigations may lead to development of clinically effective parameters that reflect NET activity. Finally, electrocardiographic and respiratory gating may be required for analyzing a moving heart. At present, we cannot expect such complete quantification using ${ }^{123} \mathrm{I}-\mathrm{mIBG}$. However, H/M ratio may be used for the initial evaluation to find the possible abnormality before proceeding to precise kinetic analysis.

\section{${ }^{123}$ I-MIBG FOR PATHOPHYSIOLOGICAL STUDIES AND PERSPECTIVES OF NET IMAGING}

${ }^{123}$ I-mIBG innervation imaging can be a powerful tool to supplement information provided by other imaging modalities. In clinical settings, clinical usefulness and effectiveness should be further validated for the evaluation of severe or lethal events including HF death, sudden cardiac death, and arrhythmic death. For physiological/pathophysiological indications, suitable reference standards are needed in order to understand the significance of ${ }^{123} \mathrm{I}-\mathrm{mIBG}$ results in populations with disease. For example, age-dependent physiological decline in ${ }^{123}$ I-mIBG uptake was observed in some studies. ${ }^{21,22}{ }^{123} \mathrm{I}$-mIBG uptake was decreased after an exposure to altitude hypoxia. ${ }^{23}$ In addition, potential influence of pre-examination medical treatments is still not confirmed systematically, except for reserpine, labetalol, and tricyclic anti-depressant. ${ }^{24}$

Within the next few years, it is likely that further research will show that genetic variations in NET coding can affect susceptibility to sympathetic nerve dysfunction, predispose patients with heart disease to develop $\mathrm{HF}$, or affect responsiveness to HF therapeutics. When such genomic discoveries are made, molecular imaging including SPECT and/or PET of NET function should be a part of the characterization of the functional significance of the genomic results. Before going to the era of generalized NET imaging, further studies on basic characteristics of ${ }^{123}$ I-mIBG are still required, even as research on new NET system radiotracers and appropriate kinetic modeling techniques continues.

\section{Disclosure}

The authors have no conflict of interest to disclose for this editorial.

\section{References}

1. Matsunari I, Aoki H, Nomura Y, Takeda N, Chen WP, Taki J, et al. Iodine-123 metaiodobenzylguanidine imaging and carbon-11 hydroxyephedrine positron emission tomography compared in patients with left ventricular dysfunction. Circ Cardiovasc Imaging. 2010;3:595-603.

2. Thackeray JT, Bengel FM. Assessment of cardiac autonomic neuronal function using PET imaging. $\mathrm{J}$ Nucl Cardiol. 2013;20:150-65.

3. Delforge J, Syrota A, Lancon JP, Nakajima K, Loc'h C, Janier M, et al. Cardiac beta-adrenergic receptor density measured in vivo using PET, CGP 12177, and a new graphical method. J Nucl Med. 1991;32:739-48.

4. Rami-Mark C, Berroteran-Infante N, Philippe C, Foltin S, Vraka C, Hoepping A, et al. Radiosynthesis and first preclinical evaluation of the novel norepinephrine transporter pet-ligand $\left[{ }^{11}\right.$ C]ME@HAPTHI. EJNMMI Res. 2015;5:113.

5. Verschure DO, Baas F, van Eck-Smit BLF, Somsen GA, Verberne HJ. Polymorphism of SLC6A2 gene does not influence outcome of myocardial ${ }^{123} \mathrm{I}-m \mathrm{IBG}$ scintigraphy in patients with chronic heart failure. J Nucl Cardiol. 2016. doi:10.1007/s12350-016-0722-x.

6. Nakajima K, Nakata T. Cardiac ${ }^{123}$ I-MIBG imaging for clinical decision making: 22-Year experience in Japan. J Nucl Med. 2015;56(Suppl 4):11S-9S.

7. Joint JCS. Working Group—guidelines for clinical use of cardiac nuclear medicine (JCS 2010)-digest version. Circ J. 2012;76:761-7.

8. Henzlova MJ, Duvall WL, Einstein AJ, Travin MI, Verberne HJ. ASNC imaging guidelines for SPECT nuclear cardiology procedures: Stress, protocols, and tracers. J Nucl Cardiol. 2016;23:60639.

9. Jacobson AF, Senior R, Cerqueira MD, Wong ND, Thomas GS, Lopez VA, et al. Myocardial iodine-123 meta-iodobenzylguanidine imaging and cardiac events in heart failure. Results of the prospective ADMIRE-HF (AdreView Myocardial Imaging for Risk Evaluation in Heart Failure) study. J Am Coll Cardiol. 2010;55:2212-21. 
10. Matsunari I, Schricke U, Bengel FM, Haase HU, Barthel P, Schmidt $\mathrm{G}$, et al. Extent of cardiac sympathetic neuronal damage is determined by the area of ischemia in patients with acute coronary syndromes. Circulation. 2000;101:2579-85.

11. Nakajima K, Yoshita M, Matsuo S, Taki J, Kinuya S. Iodine-123MIBG sympathetic imaging in Lewy-body diseases and related movement disorders. Q J Nucl Med Mol Imaging. 2008;52:37887.

12. Orimo S, Suzuki M, Inaba A, Mizusawa H. ${ }^{123}$ I-MIBG myocardial scintigraphy for differentiating Parkinson's disease from other neurodegenerative parkinsonism: A systematic review and metaanalysis. Parkinsonism Relat Disord. 2012;18:494-500.

13. McKeith I, Taylor JP, Thomas A, Donaghy P, Kane J. Revisiting DLB diagnosis: A consideration of prodromal DLB and of the diagnostic overlap with Alzheimer disease. J Geriatr Psychiatry Neurol. 2016;29:249-53.

14. Sakata K, Miura F, Sugino H, Saegusa T, Shirotani M, Yoshida H, et al. Assessment of regional sympathetic nerve activity in vasospastic angina: Analysis of iodine 123-labeled metaiodobenzylguanidine scintigraphy. Am Heart J. 1997;133:484-9.

15. Marshall A, Cheetham A, George RS, Mason M, Kelion AD. Cardiac iodine-123 metaiodobenzylguanidine imaging predicts ventricular arrhythmia in heart failure patients receiving an implantable cardioverter-defibrillator for primary prevention. Heart. 2012;98:1359-65.

16. Shannon JR, Flattem NL, Jordan J, Jacob G, Black BK, Biaggioni I, et al. Orthostatic intolerance and tachycardia associated with norepinephrine-transporter deficiency. N Engl J Med. 2000;342: 541-9.

17. Kim CH, Hahn MK, Joung Y, Anderson SL, Steele AH, MazeiRobinson MS, et al. A polymorphism in the norepinephrine transporter gene alters promoter activity and is associated with attention-deficit hyperactivity disorder. Proc Natl Acad Sci USA. 2006;103:19164-9.

18. Nakajima K, Okuda K, Yoshimura M, Matsuo S, Wakabayashi H, Imanishi $\mathrm{Y}$, et al. Multicenter cross-calibration of I-123 metaiodobenzylguanidine heart-to-mediastinum ratios to overcome camera-collimator variations. J Nucl Cardiol. 2014;21:9708 .

19. Nakajima K, Nakata T, Matsuo S, Jacobson AF. Creation of mortality risk charts using ${ }^{123}$ I meta-iodobenzylguanidine heart-tomediastinum ratio in patients with heart failure: 2- and 5-year risk models. Eur Heart J Cardiovasc Imaging. 2016;17:1138-45.

20. Giorgetti A, Burchielli S, Positano V, Kovalski G, Quaranta A, Genovesi D, et al. Dynamic 3D analysis of myocardial sympathetic innervation: An experimental study using ${ }^{123} \mathrm{I}-\mathrm{MIBG}$ and a CZT camera. J Nucl Med. 2015;56:464-9.

21. Tsuchimochi S, Tamaki N, Tadamura E, Kawamoto M, Fujita T, Yonekura $\mathrm{Y}$, et al. Age and gender differences in normal myocardial adrenergic neuronal function evaluated by iodine-123MIBG imaging. J Nucl Med. 1995;36:969-74.

22. Chen W, Botvinick EH, Alavi A, Zhang Y, Yang S, Perini R, et al. Age-related decrease in cardiopulmonary adrenergic neuronal function in children as assessed by I-123 metaiodobenzylguanidine imaging. J Nucl Cardiol. 2008;15:73-9.

23. Richalet JP, Merlet P, Bourguignon M, Le-Trong JL, Keromes A, Rathat C, et al. MIBG scintigraphic assessment of cardiac adrenergic activity in response to altitude hypoxia. $\mathrm{J}$ Nucl Med. 1990;31:34-7.

24. Jacobson AF, Travin MI. Impact of medications on mIBG uptake, with specific attention to the heart: Comprehensive review of the literature. J Nucl Cardiol. 2015;22:980-93. 